Acta Crystallographica Section E

Structure Reports

Online

ISSN 1600-5368

\section{Bis(2,6-dimethylpyridyl)iodonium dibromoiodate}

Andrei S. Batsanov, ${ }^{a}$ Andrew P. Lightfoot, ${ }^{b}$ Steven J. R. Twiddle ${ }^{a}$ and Andrew Whiting ${ }^{\mathrm{a} *}$

${ }^{a}$ Department of Chemistry, University of Durham, South Road, Durham DH1 3LE, England, and ${ }^{\mathbf{b}}$ GlaxoSmithKline

Pharmaceuticals, New Frontiers Science Park, Third Avenue, Harlow, Essex, CM19 5AW, England

Correspondence e-mail: andy.whiting@durham.ac.uk

Key indicators

Single-crystal X-ray study

$T=120 \mathrm{~K}$

Mean $\sigma(\mathrm{C}-\mathrm{C})=0.005 \AA$

$R$ factor $=0.031$

$w R$ factor $=0.076$

Data-to-parameter ratio $=27.5$

For details of how these key indicators were automatically derived from the article, see http://journals.iucr.org/e.
(C) 2006 International Union of Crystallography All rights reserved
The crystal structure of the title compound, $\mathrm{C}_{14} \mathrm{H}_{18} \mathrm{IN}_{2}{ }^{+} \cdot \mathrm{Br}_{2} \mathrm{I}^{-}$, isostructural with the $\mathrm{Cl}_{2} \mathrm{I}$ analogue, comprises discrete centrosymmetric cations and anions, both with linear coordination of the I atoms.

\section{Comment}

Electropositive sources of iodine are useful reagents for the iododeboronation of alkenylboronate derivatives (Brown et al., 1973). Iodine monochloride is an important representative of such reagents (Stewart \& Whiting, 1995; Lightfoot et al., 2004), but its shortcomings concerning reactivity, stereocontrol and chemoselectivity necessitated the development of adjusted reagents involving amine- $\mathrm{ICl}$ complexes (Batsanov et al., 2005). In the course of the latter work, we obtained bis(2,6-dimethylpyridyl)iodonium dichloroiodate (I), the crystal structure of which unexpectedly comprised discrete $\mathrm{I}\left(\mathrm{NC}_{7} \mathrm{H}_{9}\right)_{2}{ }^{+}$and $\mathrm{ICl}_{2}{ }^{-}$ions rather than neutral $\mathrm{Cl}-\mathrm{I}-\mathrm{NC}_{7} \mathrm{H}_{9}$ molecules (Batsanov et al., 2005), but which also proved to be active in iododeboronation. Continuing these studies, we have prepared the bromide analogue of compound (I), viz. $\mathrm{I}\left(\mathrm{NC}_{7} \mathrm{H}_{9}\right)_{2}{ }^{+} \cdot \mathrm{IBr}_{2}{ }^{-}$(II), which proved not to be superior to (I) as an iododeboronation agent.

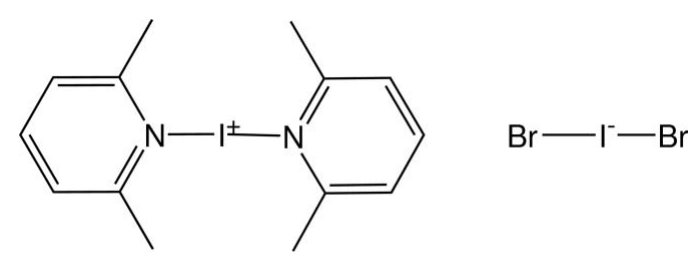

(II)

The crystals of (II) are isomorphous with those of (I), with an increase of the volume per molecule by $14 \AA^{3}$, or ca $3 \%$. The structure comprises discrete bis(2,6-dimethylpyridyl)iodonium cations and $\mathrm{IBr}_{2}{ }^{-}$anions (Fig. 1). In both ions, the central I atoms occupy special positions at inversion centres, hence the $\mathrm{N}-\mathrm{I} 1-\mathrm{N}^{\prime}$ and $\mathrm{Br}-\mathrm{I} 2-\mathrm{Br}^{\prime}$ angles exactly equal $180^{\circ}$.

Atom I1 is tilted out of the pyridine ring plane by 0.190 (5) $\AA$, whereas atoms C1 and C7 deviate on the opposite side of the plane by 0.026 (6) and 0.080 (6) $\AA$, respectively. Thus, the two rings of the cation are parallel but not coplanar, with an interplanar separation of $0.38 \AA$, $c f .0 .60 \AA$ in (I). The I1 - N bond distance of 2.294 (3) $\AA$ agrees with 2.300 (1) $\AA$ in (I), 2.259 (3) $\AA$ in bis(pyridine)iodonium (Álvarez-Rúa et al., 2002) and 2.29 (1) $\AA$ in bis(2,4,6-collidine)iodonium (Brayer $\&$ James, 1982). All these distances are much longer than the single $\mathrm{N} s p^{2}-\mathrm{I}$ bonds in $N$-iodosuccinimide [2.059 (4) $\AA$; Padmanabhan et al., 1990] or diiodoformamide [mean
Received 27 January 2006 Accepted 30 January 2006 
2.07 (3) $\AA$; Pritzkow, 1974] and can be regarded as hypervalent bonds. Likewise, the I2- Br bond length of 2.6962 (4) $\AA$ is normal for $\mathrm{IBr}_{2}{ }^{-}$anions in the solid state, $c f .2 .710$ (1) $\AA$ in $\left[\mathrm{Me}_{3} \mathrm{~S}\right]\left[\mathrm{IBr}_{2}\right]$ (Svensson \& Kloo, 2000), $2.709(2) \AA$ in $\left[\mathrm{H}_{2}(\mathrm{pc})\right]\left[\mathrm{IBr}_{2}\right]$ or $2.6986(4) \AA$ in $\left[\mathrm{H}_{2}(\mathrm{pc})\right]_{2}\left[\mathrm{IBr}_{2}\right] \mathrm{Br}$ (pc is phthalocyanine; Gardberg et al., 2002). However, these bonds also are much weaker than a single bond, as observed in the IBr molecule in the gas phase $(2.469 \AA$; Huber \& Herzberg, 1979).

\section{Experimental}

A $1.0 M$ solution of $\mathrm{IBr}$ in dichloromethane (DCM; $30 \mathrm{mmol}, 30 \mathrm{ml}$ ) was cooled to $273 \mathrm{~K}$ with stirring under argon prior to the dropwise addition of 2,6-lutidine ( $30 \mathrm{mmol}, 3.50 \mathrm{ml})$. After $30 \mathrm{~min}$, the reaction was allowed to warm to room temperature before the addition of hexane $(40 \mathrm{ml})$ to induce precipitation of the product. Filtration, drying $\left(\mathrm{MgSO}_{4}\right)$ and evaporation gave the product as an orange solid (7.37 g, 78\%). IR $v_{\max }, \mathrm{cm}^{-1}: 2978,1601,1466,1377,1161$ and 792. ${ }^{1} \mathrm{H}$ NMR (400 MHz, $\mathrm{CDCl}_{3}$, $\delta$, p.p.m.): $2.78(6 \mathrm{H}, s, 2 \times \mathrm{Me}), 7.13(2 \mathrm{H}$, $d, J=7.6 \mathrm{~Hz}, \mathrm{Ar}-\mathrm{H})$ and $7.62(1 \mathrm{H}, t, J=7.6 \mathrm{~Hz}, \mathrm{Ar}-\mathrm{H}) .{ }^{13} \mathrm{C} \mathrm{NMR}$ (100 MHz, $\mathrm{CDCl}_{3}, \delta$, p.p.m.): 28.1 (Me), 123.0 (Ar), 139.2 (Ar) and 157.6 (Ar). $\left(\mathrm{C}_{7} \mathrm{H}_{9} \mathrm{NIBr}\right)_{2}$ requires: $\mathrm{C} 26.76, \mathrm{H} 2.89, \mathrm{~N} 4.46 \%$; found: C $26.22, \mathrm{H} 2.88, \mathrm{~N} 4.28 \%$. Single crystals of X-ray quality were obtained by slow evaporation of a solution in DCM-hexane (1:1). To test the deboronation properties of (II), it has been reacted with 4,4,5,5-tetramethyl-2-non-1-enyl-1,3,2-dioxaborolane and 4,4,6trimethyl-2-non-1-enyl-1,3,2-dioxaborinane in DCM, yielding $\mathrm{BrCH}=\mathrm{CHC}_{7} \mathrm{H}_{15}$ as the sole product and with complete selectivity for the $Z$-alkene in both cases. However, the maximum conversions achieved (47 and $52 \%$, respectively) were low.

\section{Crystal data}

$$
\begin{aligned}
& \mathrm{C}_{14} \mathrm{H}_{18} \mathrm{IN}_{2}{ }^{+} \cdot \mathrm{Br}_{2} \mathrm{I}^{-} \\
& M_{r}=627.92 \\
& \text { Triclinic, } P \overline{1} \\
& a=7.5777(7) \AA \\
& b=8.2610(7) \AA \\
& c=8.5800(7) \AA \\
& \alpha=99.09(1)^{\circ} \\
& \beta=101.45(1)^{\circ} \\
& \gamma=114.20(1)^{\circ} \\
& V=462.6(1) \AA^{3}
\end{aligned}
$$

$$
\begin{aligned}
& Z=1 \\
& D_{x}=2.254 \mathrm{Mg} \mathrm{m}^{-3} \\
& \text { Mo } K \alpha \text { radiation } \\
& \text { Cell parameters from } 3235 \\
& \quad \text { reflections } \\
& \theta=2.5-30.0^{\circ} \\
& \mu=7.71 \mathrm{~mm}^{-1} \\
& T=120(2) \mathrm{K} \\
& \text { Block, orange } \\
& 0.10 \times 0.08 \times 0.02 \mathrm{~mm}
\end{aligned}
$$

\section{Data collection}

$$
\begin{array}{ll}
\text { Bruker SMART 6K CCD area- } & 2693 \text { independent reflections } \\
\text { detector diffractometer } & 2309 \text { reflections with } I>2 \sigma(I) \\
\omega \text { scans } & R_{\text {int }}=0.037 \\
\text { Absorption correction: multi-scan } & \theta_{\max }=30.0^{\circ} \\
\quad(S A D A B S ; \text { Bruker, 2003) } & h=-10 \rightarrow 10 \\
T_{\min }=0.637, T_{\max }=0.861 & k=-11 \rightarrow 11 \\
6548 \text { measured reflections } & l=-12 \rightarrow 12
\end{array}
$$

\section{Refinement}

Refinement on $F^{2}$

$R\left[F^{2}>2 \sigma\left(F^{2}\right)\right]=0.031$

$w R\left(F^{2}\right)=0.076$

$S=0.99$

2693 reflections

98 parameters
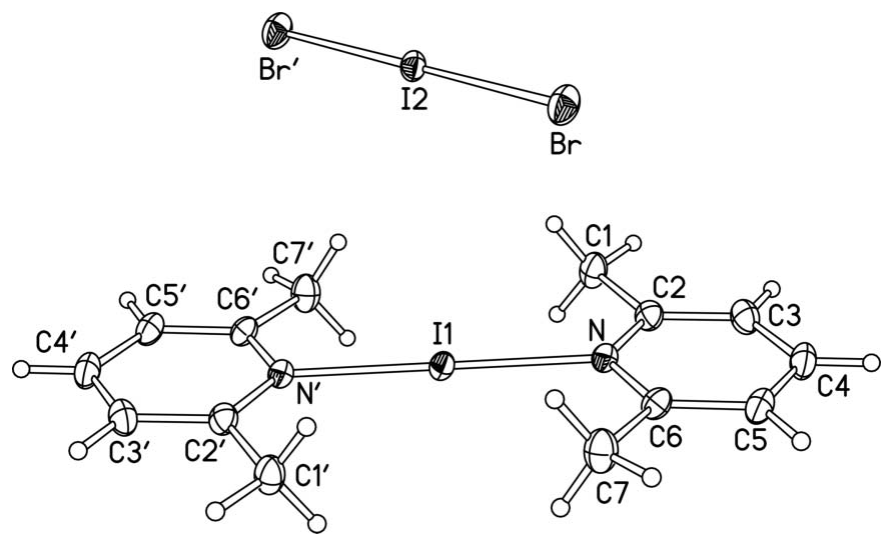

Figure 1

The cation and anion in (II). Atomic displacement ellipsoids are drawn at the $50 \%$ probability level. $\mathrm{Br}^{\prime}$ is generated by the symmetry operator $(-x$, $-y, 1-z)$, and the other primed atoms are generated by the symmetry operator $(1-x, 1-y, 1-z)$.

Methyl groups were treated as rigid bodies $(\mathrm{C}-\mathrm{H}=0.98 \AA)$ rotating around the $\mathrm{C}-\mathrm{C}$ bonds, with a common refined $U_{\text {iso }}$ value for three $\mathrm{H}$ atoms. Aromatic $\mathrm{H}$ atoms were treated as riding on the $\mathrm{C}$ atoms $\left[\mathrm{C}-\mathrm{H}=0.95 \AA\right.$ and $\left.U_{\text {iso }}(\mathrm{H})=1.2 U_{\text {eq }}(\mathrm{C})\right]$. The five strongest maxima and minima of the final difference map are located at distances of $0.8-0.9 \AA$ from atoms I1 and I2.

Data collection: SMART (Bruker, 2001); cell refinement: SAINT (Bruker, 2001); data reduction: $S A I N T$; $\operatorname{program}(\mathrm{s})$ used to solve structure: SHELXTL (Bruker, 2001); program(s) used to refine structure: SHELXTL; molecular graphics: SHELXTL; software used to prepare material for publication: SHELXTL.

The authors are grateful to the EPSRC for a DTA award to SJRT and to GlaxoSmithKline Pharmaceuticals for a CASE studentship.

\section{References}

Álvarez-Rúa, C., García-Granda, S., Bellesteros, A., Gonzáles-Bobes, F. \& Gonzáles, J. M. (2002). Acta Cryst. E58, o1381-o1383.

Batsanov, A. S., Howard, J. A. K., Lightfoot, A. P., Twiddle, S. J. R. \& Whiting, A. (2005). Eur. J. Org. Chem. pp. 1876-1883.

Brayer, G. D. \& James, M. N. G. (1982). Acta Cryst. B38, 654-657.

Brown H. C., Hamaoka T. \& Ravindran N. (1973). J. Am. Chem. Soc. 95, 57865788.

Bruker (2001). SMART (Version 5.625), SAINT (Version 6.02A) and SHELXTL (Version 6.12). Bruker AXS Inc, Madison, Wisconsin, USA.

Bruker (2003). SADABS. Version 2.10. Bruker AXS Inc, Madison, Wisconsin, USA.

Gardberg, A. S., Yang, S., Hoffman, B. M. \& Ibers, J. A. (2002). Inorg. Chem. 41, 1778-1781.

Huber, K. P. \& Herzberg, G. (1979). In Molecular Spectra and Molecular Structure, Vol. IV, Constants of Diatomic Molecules. New York: van Nostrand.

Lightfoot, A. P., Twiddle, S. J. R. \& Whiting, A. (2004). Tetrahedron Lett. 45, 8557-8561.

Padmanabhan, K., Paul, I. C. \& Curtin, D. Y. (1990). Acta Cryst. C46, 88-92. Pritzkow, H. (1974). Monatsh. Chem. 105, 621-628.

Stewart, S. K. \& Whiting A. (1995). Tetrahedron Lett. 36, 3929-3932.

Svensson, P. H. \& Kloo, L. (2000). J. Chem. Soc. Dalton Trans. pp. 2449-2455. 


\section{supporting information}

Acta Cryst. (2006). E62, o901-o902 [https://doi.org/10.1107/S1600536806003680]

\section{Bis(2,6-dimethylpyridyl)iodonium dibromoiodate}

\section{Andrei S. Batsanov, Andrew P. Lightfoot, Steven J. R. Twiddle and Andrew Whiting}

Bis(2,6-dimethylpyridyl)iodinium dibromoiodate

Crystal data

$\mathrm{C}_{14} \mathrm{H}_{18} \mathrm{IN}_{2}^{+} \cdot \mathrm{Br}_{2} \mathrm{I}^{-}$

$M_{r}=627.92$

Triclinic, $P \overline{1}$

$a=7.5777(7) \AA$

$b=8.2610(7) \AA$

$c=8.5800(7) \AA$

$\alpha=99.09(1)^{\circ}$

$\beta=101.45(1)^{\circ}$

$\gamma=114.20(1)^{\circ}$

$V=462.6(1) \AA^{3}$

$Z=1$

\section{Data collection}

Bruker SMART 6K CCD area-detector diffractometer

Radiation source: fine-focus sealed tube Graphite monochromator

Detector resolution: 5.6 pixels $\mathrm{mm}^{-1}$

$\omega$ scans

Absorption correction: multi-scan

(SADABS; Bruker, 2003)

$T_{\text {min }}=0.637, T_{\max }=0.861$

Refinement

Refinement on $F^{2}$

Least-squares matrix: full

$R\left[F^{2}>2 \sigma\left(F^{2}\right)\right]=0.031$

$w R\left(F^{2}\right)=0.076$

$S=0.99$

2693 reflections

98 parameters

0 restraints

Primary atom site location: structure-invariant direct methods
$F(000)=292$

$D_{\mathrm{x}}=2.254 \mathrm{Mg} \mathrm{m}^{-3}$

Melting point: $383(1) \mathrm{K}$

Mo $K \alpha$ radiation, $\lambda=0.71073 \AA$

Cell parameters from 3235 reflections

$\theta=2.5-30.0^{\circ}$

$\mu=7.71 \mathrm{~mm}^{-1}$

$T=120 \mathrm{~K}$

Block, orange

$0.10 \times 0.08 \times 0.02 \mathrm{~mm}$

6548 measured reflections

2693 independent reflections

2309 reflections with $I>2 \sigma(I)$

$R_{\text {int }}=0.037$

$\theta_{\max }=30.0^{\circ}, \theta_{\min }=2.5^{\circ}$

$h=-10 \rightarrow 10$

$k=-11 \rightarrow 11$

$l=-12 \rightarrow 12$

Secondary atom site location: difference Fourier map

Hydrogen site location: inferred from neighbouring sites

$\mathrm{H}$-atom parameters constrained

$w=1 /\left[\sigma^{2}\left(F_{0}^{2}\right)+(0.0387 P)^{2}\right]$

where $P=\left(F_{\mathrm{o}}{ }^{2}+2 F_{\mathrm{c}}{ }^{2}\right) / 3$

$(\Delta / \sigma)_{\max }<0.001$

$\Delta \rho_{\max }=2.54 \mathrm{e} \AA^{-3}$

$\Delta \rho_{\min }=-1.41$ e $\AA^{-3}$

Special details

Experimental. The data collection nominally covered full sphere of reciprocal space, by a combination of 3 sets of $\omega$ scans; each set at different $\varphi$ angles and each scan (20 s exposure) covering $0.3^{\circ}$ in $\omega$. Crystal to detector distance 4.85 $\mathrm{cm}$. 
Geometry. All e.s.d.'s (except the e.s.d. in the dihedral angle between two 1.s. planes) are estimated using the full covariance matrix. The cell e.s.d.'s are taken into account individually in the estimation of e.s.d.'s in distances, angles and torsion angles; correlations between e.s.d.'s in cell parameters are only used when they are defined by crystal symmetry. An approximate (isotropic) treatment of cell e.s.d.'s is used for estimating e.s.d.'s involving 1.s. planes.

Refinement. Refinement of $F^{2}$ against ALL reflections. The weighted $R$-factor $w R$ and goodness of fit $S$ are based on $F^{2}$, conventional $R$-factors $R$ are based on $F$, with $F$ set to zero for negative $F^{2}$. The threshold expression of $F^{2}>\sigma\left(F^{2}\right)$ is used only for calculating $R$-factors(gt) etc. and is not relevant to the choice of reflections for refinement.

Fractional atomic coordinates and isotropic or equivalent isotropic displacement parameters $\left(\AA^{2}\right)$

\begin{tabular}{lllll}
\hline & $x$ & $y$ & $z$ & $U_{\text {iso }} / U_{\text {eq }}$ \\
\hline I1 & 0.5000 & 0.5000 & 0.5000 & $0.01576(9)$ \\
$\mathrm{I} 2$ & 0.0000 & 0.0000 & 0.5000 & $0.01764(9)$ \\
$\mathrm{Br}$ & $-0.12080(6)$ & $0.18586(6)$ & $0.30774(5)$ & $0.02574(10)$ \\
$\mathrm{N}$ & $0.3548(4)$ & $0.4793(4)$ & $0.2315(3)$ & $0.0157(5)$ \\
$\mathrm{C} 1$ & $0.3316(6)$ & $0.1696(5)$ & $0.1580(5)$ & $0.0237(8)$ \\
$\mathrm{H} 11$ & 0.4749 & 0.2085 & 0.2109 & $0.028(7)^{*}$ \\
$\mathrm{H} 12$ & 0.2822 & 0.0684 & 0.0584 & $0.028(7)^{*}$ \\
$\mathrm{H} 13$ & 0.2535 & 0.1279 & 0.2347 & $0.028(7)^{*}$ \\
$\mathrm{C} 2$ & $0.3060(5)$ & $0.3288(5)$ & $0.1115(4)$ & $0.0186(7)$ \\
$\mathrm{C} 3$ & $0.2344(5)$ & $0.3203(5)$ & $-0.0527(4)$ & $0.0225(7)$ \\
$\mathrm{H} 3$ & 0.2021 & 0.2140 & -0.1366 & $0.027^{*}$ \\
$\mathrm{C} 4$ & $0.2092(6)$ & $0.4668(6)$ & $-0.0944(4)$ & $0.0230(7)$ \\
$\mathrm{H} 4$ & 0.1604 & 0.4626 & -0.2066 & $0.028^{*}$ \\
$\mathrm{C} 5$ & $0.2572(5)$ & $0.6189(5)$ & $0.0308(4)$ & $0.0210(7)$ \\
$\mathrm{H} 5$ & 0.2416 & 0.7207 & 0.0046 & $0.025^{*}$ \\
$\mathrm{C} 6$ & $0.3291(5)$ & $0.6239(5)$ & $0.1946(4)$ & $0.0173(6)$ \\
$\mathrm{C} 7$ & $0.3721(6)$ & $0.7836(6)$ & $0.3326(5)$ & $0.0256(8)$ \\
$\mathrm{H} 71$ & 0.2964 & 0.7385 & 0.4099 & $0.033(7)^{*}$ \\
$\mathrm{H} 72$ & 0.3305 & 0.8685 & 0.2873 & $0.033(7)^{*}$ \\
$\mathrm{H} 73$ & 0.5173 & 0.8483 & 0.3908 & $0.033(7)^{*}$ \\
& & & & \\
\hline
\end{tabular}

Atomic displacement parameters $\left(\AA^{2}\right)$

\begin{tabular}{lllllll}
\hline & $U^{11}$ & $U^{22}$ & $U^{33}$ & $U^{12}$ & $U^{13}$ & $U^{23}$ \\
\hline $\mathrm{I} 1$ & $0.01858(15)$ & $0.01675(16)$ & $0.01325(15)$ & $0.00838(12)$ & $0.00566(11)$ & $0.00521(11)$ \\
$\mathrm{I} 2$ & $0.01924(16)$ & $0.02170(17)$ & $0.01317(15)$ & $0.01004(13)$ & $0.00530(11)$ & $0.00509(12)$ \\
$\mathrm{Br}$ & $0.0292(2)$ & $0.0335(2)$ & $0.02382(19)$ & $0.01926(17)$ & $0.01002(15)$ & $0.01507(16)$ \\
$\mathrm{N}$ & $0.0171(13)$ & $0.0173(14)$ & $0.0139(13)$ & $0.0083(11)$ & $0.0056(10)$ & $0.0051(11)$ \\
$\mathrm{C} 1$ & $0.036(2)$ & $0.0186(18)$ & $0.0163(17)$ & $0.0139(16)$ & $0.0061(15)$ & $0.0035(14)$ \\
$\mathrm{C} 2$ & $0.0186(16)$ & $0.0225(18)$ & $0.0160(16)$ & $0.0097(14)$ & $0.0073(13)$ & $0.0051(13)$ \\
$\mathrm{C} 3$ & $0.0237(18)$ & $0.026(2)$ & $0.0155(16)$ & $0.0114(15)$ & $0.0044(13)$ & $0.0023(14)$ \\
$\mathrm{C} 4$ & $0.0255(18)$ & $0.031(2)$ & $0.0155(16)$ & $0.0143(16)$ & $0.0065(14)$ & $0.0100(15)$ \\
$\mathrm{C} 5$ & $0.0235(17)$ & $0.0264(19)$ & $0.0196(17)$ & $0.0141(15)$ & $0.0087(14)$ & $0.0123(15)$ \\
$\mathrm{C} 6$ & $0.0201(16)$ & $0.0201(17)$ & $0.0163(16)$ & $0.0107(14)$ & $0.0101(13)$ & $0.0065(13)$ \\
$\mathrm{C} 7$ & $0.033(2)$ & $0.028(2)$ & $0.0201(18)$ & $0.0191(17)$ & $0.0072(15)$ & $0.0059(15)$ \\
\hline
\end{tabular}


Geometric parameters $\left(\AA,{ }^{o}\right)$

\begin{tabular}{|c|c|c|c|}
\hline $\mathrm{I} 1-\mathrm{N}^{\mathrm{i}}$ & $2.294(3)$ & $\mathrm{C} 3-\mathrm{C} 4$ & $1.389(5)$ \\
\hline $\mathrm{I} 1-\mathrm{N}$ & $2.294(3)$ & $\mathrm{C} 3-\mathrm{H} 3$ & 0.9500 \\
\hline $\mathrm{I} 2-\mathrm{Br}$ & $2.6962(4)$ & $\mathrm{C} 4-\mathrm{C} 5$ & $1.382(5)$ \\
\hline $\mathrm{I} 2-\mathrm{Br}^{\mathrm{ri}}$ & $2.6962(4)$ & $\mathrm{C} 4-\mathrm{H} 4$ & 0.9500 \\
\hline $\mathrm{N}-\mathrm{C} 2$ & $1.345(5)$ & $\mathrm{C} 5-\mathrm{C} 6$ & $1.390(5)$ \\
\hline $\mathrm{N}-\mathrm{C} 6$ & $1.361(4)$ & $\mathrm{C} 5-\mathrm{H} 5$ & 0.9500 \\
\hline $\mathrm{C} 1-\mathrm{C} 2$ & $1.507(5)$ & $\mathrm{C} 6-\mathrm{C} 7$ & $1.500(5)$ \\
\hline $\mathrm{C} 1-\mathrm{H} 11$ & 0.9801 & $\mathrm{C} 7-\mathrm{H} 71$ & 0.9800 \\
\hline $\mathrm{C} 1-\mathrm{H} 12$ & 0.9800 & $\mathrm{C} 7-\mathrm{H} 72$ & 0.9800 \\
\hline $\mathrm{C} 1-\mathrm{H} 13$ & 0.9800 & $\mathrm{C} 7-\mathrm{H} 73$ & 0.9801 \\
\hline $\mathrm{C} 2-\mathrm{C} 3$ & $1.385(5)$ & & \\
\hline $\mathrm{N}^{\mathrm{i}}-\mathrm{I} 1-\mathrm{N}$ & 180.0 & $\mathrm{C} 4-\mathrm{C} 3-\mathrm{H} 3$ & 120.1 \\
\hline $\mathrm{Br}-\mathrm{I} 2-\mathrm{Br}^{\mathrm{ri}}$ & $179.999(12)$ & $\mathrm{C} 5-\mathrm{C} 4-\mathrm{C} 3$ & $118.6(3)$ \\
\hline $\mathrm{C} 2-\mathrm{N}-\mathrm{C} 6$ & $120.8(3)$ & $\mathrm{C} 5-\mathrm{C} 4-\mathrm{H} 4$ & 120.8 \\
\hline $\mathrm{C} 2-\mathrm{N}-\mathrm{I} 1$ & $119.8(2)$ & $\mathrm{C} 3-\mathrm{C} 4-\mathrm{H} 4$ & 120.7 \\
\hline $\mathrm{C} 6-\mathrm{N}-\mathrm{I} 1$ & $119.4(2)$ & $\mathrm{C} 4-\mathrm{C} 5-\mathrm{C} 6$ & $120.3(3)$ \\
\hline $\mathrm{C} 2-\mathrm{C} 1-\mathrm{H} 11$ & 109.7 & $\mathrm{C} 4-\mathrm{C} 5-\mathrm{H} 5$ & 119.7 \\
\hline $\mathrm{C} 2-\mathrm{C} 1-\mathrm{H} 12$ & 109.3 & $\mathrm{C} 6-\mathrm{C} 5-\mathrm{H} 5$ & 120.0 \\
\hline $\mathrm{H} 11-\mathrm{C} 1-\mathrm{H} 12$ & 109.5 & $\mathrm{~N}-\mathrm{C} 6-\mathrm{C} 5$ & $119.8(3)$ \\
\hline $\mathrm{C} 2-\mathrm{C} 1-\mathrm{H} 13$ & 109.4 & $\mathrm{~N}-\mathrm{C} 6-\mathrm{C} 7$ & $119.1(3)$ \\
\hline $\mathrm{H} 11-\mathrm{C} 1-\mathrm{H} 13$ & 109.5 & $\mathrm{C} 5-\mathrm{C} 6-\mathrm{C} 7$ & $121.1(3)$ \\
\hline $\mathrm{H} 12-\mathrm{C} 1-\mathrm{H} 13$ & 109.5 & $\mathrm{C} 6-\mathrm{C} 7-\mathrm{H} 71$ & 109.5 \\
\hline $\mathrm{N}-\mathrm{C} 2-\mathrm{C} 3$ & $120.7(3)$ & $\mathrm{C} 6-\mathrm{C} 7-\mathrm{H} 72$ & 109.4 \\
\hline $\mathrm{N}-\mathrm{C} 2-\mathrm{C} 1$ & 119.0 & $\mathrm{H} 71-\mathrm{C} 7-\mathrm{H} 72$ & 109.5 \\
\hline $\mathrm{C} 3-\mathrm{C} 2-\mathrm{C} 1$ & $120.3(3)$ & $\mathrm{C} 6-\mathrm{C} 7-\mathrm{H} 73$ & 109.6 \\
\hline $\mathrm{C} 2-\mathrm{C} 3-\mathrm{C} 4$ & $119.9(3)$ & $\mathrm{H} 71-\mathrm{C} 7-\mathrm{H} 73$ & 109.5 \\
\hline $\mathrm{C} 2-\mathrm{C} 3-\mathrm{H} 3$ & 120.0 & $\mathrm{H} 72-\mathrm{C} 7-\mathrm{H} 73$ & 109.5 \\
\hline
\end{tabular}

Symmetry codes: (i) $-x+1,-y+1,-z+1$; (ii) $-x,-y,-z+1$. 